\title{
Angle-resolved cathodoluminescence spectroscopy
}

\author{
Toon Coenen, ${ }^{\text {a) }}$ Ernst Jan R. Vesseur, and Albert Polman \\ Center for Nanophotonics, FOM Institute AMOLF, Science Park 104, 1098 XG Amsterdam, The Netherlands
}

(Received 18 July 2011; accepted 10 September 2011; published online 4 October 2011)

\begin{abstract}
We present a cathodoluminescence spectroscopy technique which combines deep subwavelength excitation resolution with angle-resolved detection capabilities. The cathodoluminescence emission is collected by a paraboloid mirror (effective $\mathrm{NA}=0.96$ ) and is projected onto a $2 \mathrm{D} \mathrm{CCD}$ array. The azimuthal and polar emission pattern is directly deduced from the image. As proof of principle, we use the technique to measure the angular distribution of transition radiation from a single crystalline gold surface under $30 \mathrm{keV}$ electron irradiation. We find that the experiment matches very well with theory, illustrating the potential of this technique for the characterization of photonic structures with deep subwavelength dimensions. (C) 2011 American Institute of Physics. [doi:10.1063/1.3644985]
\end{abstract}

In nanophotonics, the research field concerned with the manipulation of light at the nanoscale, basic building blocks like waveguides, ${ }^{1}$ nanoantennas, ${ }^{2}$ and nanocavities, ${ }^{3}$ often have subwavelength geometrical features. Optical phenomena in these structures cannot be resolved with far-field optical microscopy due to Abbe's law of diffraction that provides a limit to the smallest distance over which individual objects can be distinguished. Several spatially resolved spectroscopy techniques have been developed to reach a resolution below that limit, including scanning near-field optical microscopy (SNOM) ${ }^{4}$ stimulated emission depletion (STED) microscopy, ${ }^{5}$ photoactivatable localization microscopy (PALM) ${ }^{6}$ and stochastic optical reconstruction microscopy (STORM). ${ }^{7}$

Cathodoluminescence (CL) imaging spectroscopy is a technique that has even higher spatial resolution, as it uses an electron beam as an excitation source. In CL-spectroscopy, an electron beam focused to a $1-10 \mathrm{~nm}$ spot is raster scanned over a sample. The electron beam effectively acts as a supercontinuum light source that excites the sample according to the local density of optical states (LDOS). ${ }^{8,9}$ Previous work has shown that CL imaging spectroscopy is a powerful technique for probing (nano)photonic environments. In particular, in the field of plasmonics, where large spatial variations in the LDOS can occur on deep subwavelength length scales, CL-spectroscopy has proven to be an important characterization technique., ${ }^{2,10,11}$

So far, the CL technique was limited to measuring spectral response as a function of excitation position, and no information about the total angular distribution of the CL emission could be collected. Yamamoto and coworkers developed a technique by which the angular distribution of $\mathrm{CL}$ was measured by translating a pinhole in front of a CCD camera, thus collecting point-by-point angular data. ${ }^{12,13}$ In this letter, we present a further advanced experimental technique to resolve the angular distribution of CL emission, in both the azimuthal and polar directions. We demonstrate the technique by measuring transition radiation (TR) for a single crystal gold substrate.

\footnotetext{
${ }^{\text {a) }}$ Author to whom correspondence should be addressed. Electronic mail: coenen@amolf.nl.
}

Figure 1(a) shows a schematic overview of the experimental setup that uses a FEI XL-30 SFEG scanning electron microscope (SEM). For CL experiments, a diamond-turned aluminum off-axis paraboloid mirror with a focal distance of $0.5 \mathrm{~mm}$ is mounted in the vacuum chamber of the microscope. Directly above its focal point, this paraboloid has a $600-\mu \mathrm{m}-$ diameter hole through which a $30 \mathrm{keV}$ electron beam can reach the sample. The CL emission is collected by the mirror and redirected out of the SEM through a glass vacuum flange. The beam emanating from the paraboloid is projected onto a Peltier-cooled $\left(-70^{\circ} \mathrm{C}\right) 1024 \times 1024$ pixels back-illuminated silicon CCD camera. An achromatic lens (focal distance $11 \mathrm{~cm}$ ) ensures that the beam fully fills the CCD array. Color filters and polarizers can be put in the beam path to add wavelength and polarization sensitivity to the setup.

For optimum detection efficiency, the electron beam must hit the sample in the focus of the mirror which requires accurate positioning of the mirror relative to the sample. The vertical distance between sample and mirror can be adjusted accurately with the SEM stage. Micro positioning of the mirror is done by four piezoelectric stepper motors integrated

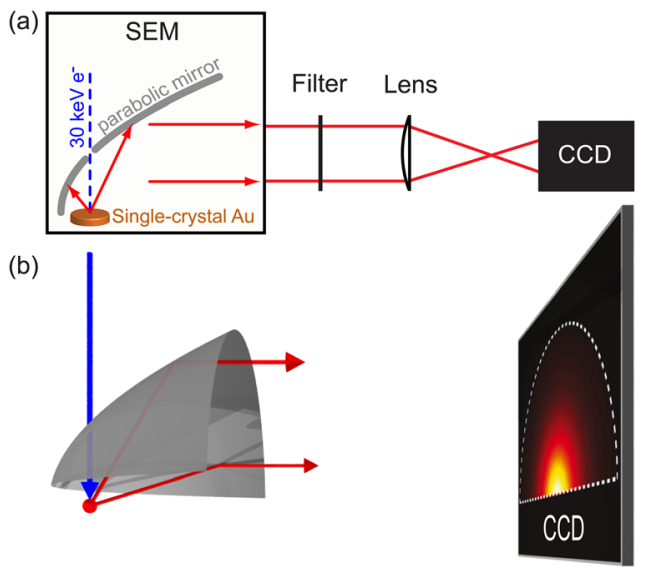

FIG. 1. (Color online) (a) Schematic overview of the experimental setup. The scanning electron microscope contains the paraboloid mirror which collects the CL-emission and redirects it towards the CCD detector. The $30 \mathrm{keV}$ electron beam reaches the sample through a hole in the mirror. The lens in the setup projects the output on a $1024 \times 1024$ pixel CCD camera. From the image, the angular distribution of cathodoluminescence emission is directly determined. (b) 3D representation of how the paraboloid beam is projected onto the CCD array for an isotropic light source. 
with a titanium leaf spring mechanism to which the mirror is attached, allowing translational (x,y) and rotational positioning (tilt and yaw) of the mirror. If the mirror is well-focused, each pixel in the CCD image of the beam corresponds to a single point on the paraboloid. Furthermore, each point in the paraboloid is associated with a unique emission angle which can be described by a zenithal angle $\theta$ running from $0^{\circ}$ to $90^{\circ}$ (where $\theta=0$ is normal to the surface) and an azimuthal angle $\varphi$ running from 0 to $360^{\circ}$. This collection geometry is very similar to a Fourier microscope where the back aperture of a microscope objective is imaged with a camera to determine angular radiation patterns. ${ }^{5}$ Figures 2(a) and 2(b) show how each pixel in the image is mapped onto angles $\theta$ and $\varphi$, calculated using the known geometrical properties of the paraboloid. Figure 2(c) shows the solid angle covered per pixel which is used to correct the data to photon flux per unit of solid angle. Integrating over all pixels that fall within the beam gives a total acceptance angle of $1.46 \pi \mathrm{sr}$, equivalent to a $\mathrm{NA}=0.96$ for a microscope objective. Taking only the half space left of the electron beam (see Fig. 1), the solid angle is $0.96 \pi$ sr, i.e., $96 \%$ of the radiation; the remaining $4 \%$ leaves through the small opening between mirror and sample. Since we have the ability to rotate the sample by $180^{\circ}$, we can almost entirely reconstruct the emission pattern for the upper hemisphere, corresponding to an NA of 0.999 .

When an electron passes through an interface between two dielectric environments, it induces a dipole moment on the surface which radiates into the far field (TR). The TR radiation pattern is very similar to that of a vertical point dipole on a surface. ${ }^{8}$ To test the angle-resolving capabilities of the setup, we measured the TR emission from a polished single-crystalline $\mathrm{Au}$ (100) surface irradiated with $30 \mathrm{keV}$ electrons over the full spectral band of the CCD. A CCD image is recorded with a collection time of only $10 \mathrm{~s}$. For each measurement, we collected an additional image with the electron beam blanked as a reference. Subsequently, this signal was subtracted from the TR-measurement to remove dark counts from the CCD and signal originating from other sources than the sample.

Figure 2(d) shows a CCD image obtained using the method described above. The semicircular shape of the paraboloid end face can clearly be recognized. The dark concentric rings in the image are caused by radial imperfections in the mirror which are inherent to the diamond turning fabrication process of the mirror. Also, the hole in the mirror is visible close to the center of the beam. The area around the hole corresponds to emission angles that are almost normal to the surface. This area is relatively dark, while other parts corresponding to more grazing angles appear to be brighter (see Fig. 2(a)). This particular pattern in the CCD image points towards a toroidally shaped emission pattern as predicted for transition radiation.

Figure 3 shows the probability of exciting a TR photon on a gold surface integrated over the entire upper hemisphere, which was calculated using the theory described in Ref. 9 and tabulated optical constants. ${ }^{14}$ Broadband emission is expected in the $350-950 \mathrm{~nm}$ spectral band, with a dip around $500 \mathrm{~nm}$ which is related to the plasmon resonance frequency in gold. Integrating the probability of TR emission from 350 to $950 \mathrm{~nm}$, we find a generation rate of $1 \times 10^{-4}$
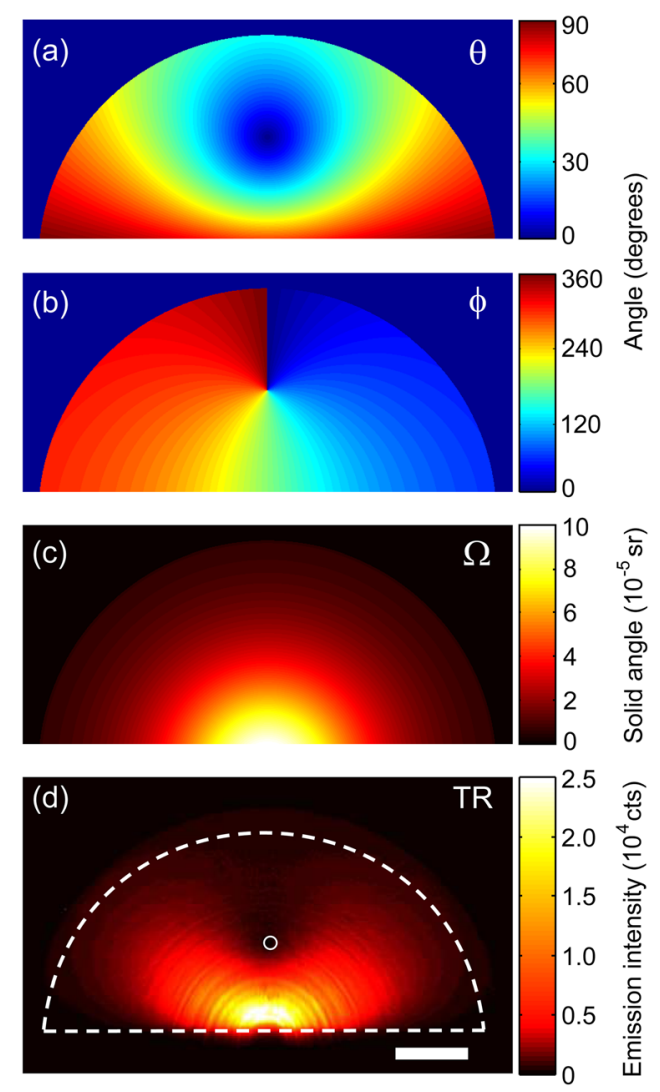

FIG. 2. (Color online) Maps relating pixel position in the CCD image to (a) polar angle $\theta$, (b) azimuthal angle $\varphi$, and (c) the solid angle per pixel, allowing conversion to an absolute radiation pattern. (d) CCD image showing the measured transition radiation from a single-crystalline gold sample. The white dashed line indicates the mirror contour, and the white circle indicates the position of the hole in the mirror. The scale bar is $2 \mathrm{~mm}$ which corresponds to $154 \mathrm{CCD}$ pixels.

photons per incident electron. In this experiment, a current of $12 \mathrm{nA}$ was used which should then generate $8 \times 10^{6}$ photons $\mathrm{s}^{-1}$. Taking into account the acceptance angle of the mirror, known reflection losses in the optics, and the quantum efficiency of the CCD camera, we expect $\sim 4 \times 10^{6}$ photons $\mathrm{s}^{-1}$ to be detected by the CCD camera. Integrating over all pixels in Fig. 2(d), we find a collection rate of $3.9 \times 10^{6}$ photons $\mathrm{s}^{-1}$; similar to the calculated value. This indicates that the angle-resolved CL technique provides an accurate absolute measurement of the emitted radiation intensity.

To investigate the directionality of the emission in more detail, the CCD image in Fig. 2(d) was converted into a radiation pattern using the conversion maps shown in Figs. 2(a)-2(c). The data were interpolated to yield points that are equally spaced in $\theta$ and $\varphi$. Figure 4(a) shows the angular distribution of TR emission mapped onto a polar grid where the radius represents polar angle $\theta$, and the polar plot angle represents azimuthal angle $\varphi$. Fig. 4(b) shows the calculated angular distribution for $30 \mathrm{keV}$ electron beam excitation integrated over the spectral sensitivity band of the CCD camera $(350-950 \mathrm{~nm})$. Data for angles that are not collected by the mirror are set to zero (black). CL emission close to the surface normal (around $\theta=0^{\circ}$ ) is not collected because of the hole in the mirror. Radiation emitted almost grazing to the surface $\left(\theta>85^{\circ}\right)$ is also not collected because of the small space between mirror and sample $(0.5 \mathrm{~mm})$. The largest loss 


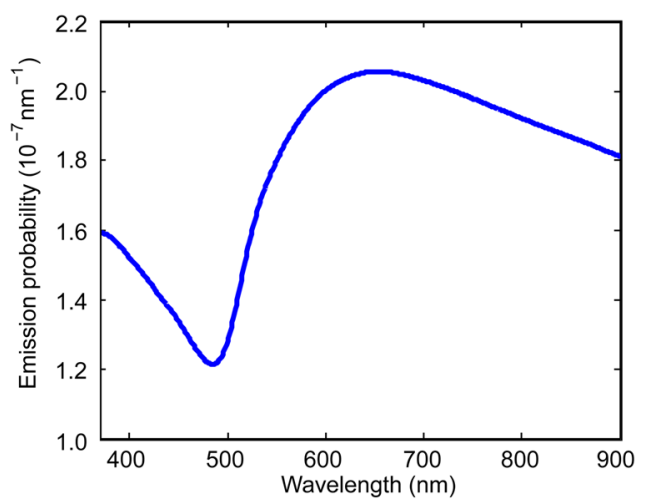

FIG. 3. (Color online) TR emission probability per incoming $30 \mathrm{keV}$ electron per nm bandwidth as a function of wavelength for the entire upper hemisphere calculated for a gold substrate.

in acceptance angle, clearly visible in the upper part of Figs. 4(a) and 4(b), corresponds to the open part of the paraboloid (see Fig. 1(b)). The experiment clearly shows a toroidal emission pattern, in good agreement with calculation. Indeed, the radiation pattern is very similar to that of a verti-
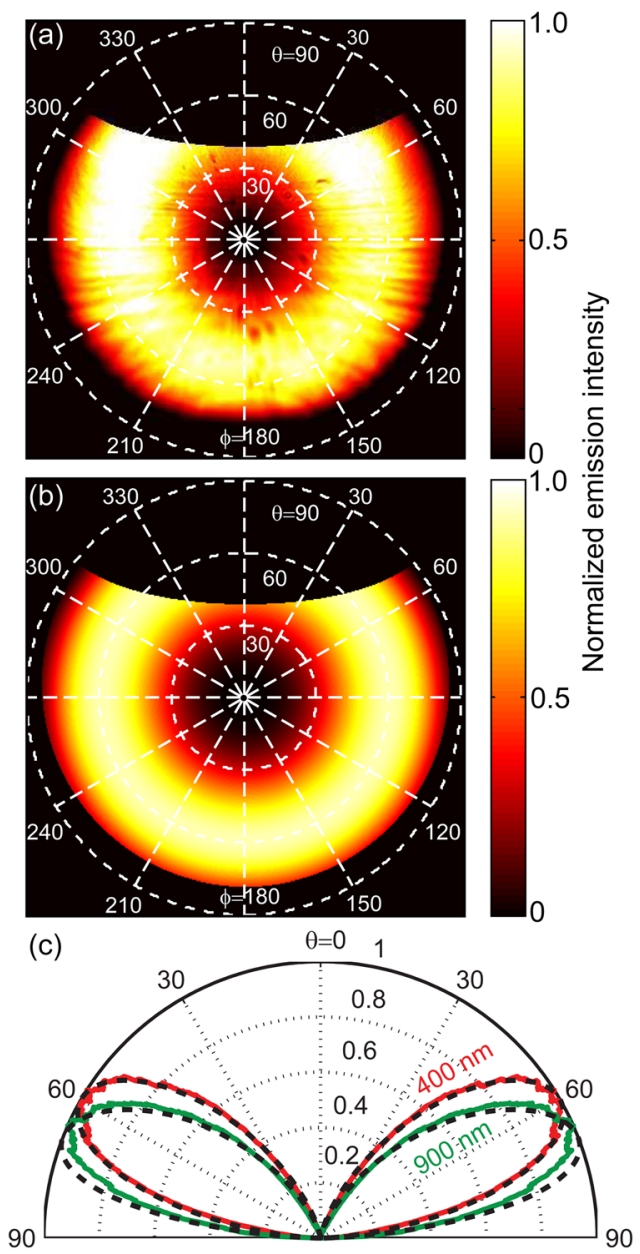

FIG. 4. (Color online) Angle-resolved cathodoluminescence emission patterns of the transition radiation for single-crystalline $\mathrm{Au}\left(30 \mathrm{keV} \mathrm{e}^{-}\right)$. (a) Normalized measured emission pattern collected without color filter showing the total emission intensity $(350-950 \mathrm{~nm})$ as a function of emission angles $\theta$ and $\varphi$. (b) Normalized calculated emission pattern for the same spectral range as in (a). (c) Emission pattern integrated over $\varphi$ for transition radiation at 400 and $900 \mathrm{~nm}$ (solid lines) and theory (dashed lines). cally oriented point dipole located close to a reflective surface. $^{4}$

Next, we investigate the wavelength dependence of the angular radiation pattern. Band pass filters $(40 \mathrm{~nm})$ were placed in the beam path with center wavelengths ranging from $400 \mathrm{~nm}$ to $900 \mathrm{~nm}$ in steps of $50 \mathrm{~nm}$, and emission patterns were measured at each wavelength. For these measurements, an integration time of $120 \mathrm{~s}$ was used to enable accurate measurements at $900 \mathrm{~nm}$ where the sensitivity of the CCD detector is lowest. To accurately compare experiment and theory, the emission patterns were integrated over azimuthal angle $\varphi$. Data in the range $\varphi=150^{\circ}-210^{\circ}$ were not taken into account as this corresponds to the section where the aberrations in the mirror are largest (see Fig. 4(a)). Figure 4(c) shows the normalized CL intensity as function of $\theta$ for $400 \mathrm{~nm}$ and $900 \mathrm{~nm}$ together with theoretically predicted emission patterns. We find excellent agreement between experiment and theory. The overall shape of the lobes is well reproduced, and the small difference in lobe orientation between these wavelengths (peak emission around $\theta=60^{\circ}$ for $400 \mathrm{~nm}$ and around $\theta=70^{\circ}$ for $900 \mathrm{~nm}$ ) is clearly observed.

In conclusion, we have presented an experimental technique for angle-resolved cathodoluminescence spectroscopy. We determined the angular distribution of transition radiation from a single crystal gold substrate and found that the experimental results agree very well with theory. A photon count rate in the order of $10^{6}$ photons $\mathrm{s}^{-1}$ is observed, corresponding closely to the calculated value. The mirror collection geometry corresponds to an effective $\mathrm{NA}=0.96$. Combining the angle resolved capabilities with the high spatial excitation resolution of electron microscopy makes this cathodoluminescence technique an interesting tool for studying a wealth of optical phenomena in a wide variety of photonic nanostructures.

We would like to thank Hans Zeijlemaker, Iliya Cerjak, and Johan Derks for their technical support. This work is a part of the research program of the "Stichting voor Fundamenteel Onderzoek der Materie (FOM)," which is financially supported by the "Nederlandse Organisatie voor Wetenschappelijk Onderzoek (NWO)." This work is also a part of NanoNextNL, a nanotechnology program funded by the Dutch ministry of economic affairs.

${ }^{1}$ E. Verhagen, M. Spasenović, A. Polman, and L. Kuipers, Phys. Rev. Lett. 102, 203904 (2009).

${ }^{2}$ E. J. R. Vesseur, J. García de Abajo, and A. Polman, Nano Lett. 9, 3147 (2009).

${ }^{3}$ L. Novotny and N. F. van Hulst, Nat. Photonics 5, 83 (2011).

${ }^{4}$ L. Novotny and B. Hecht, Principles of Nano-Optics (Cambridge University press, New York, 2006).

${ }^{5}$ V. Westphal and S. W. Hell, Phys. Rev. Lett. 94, 143903 (2005).

${ }^{6}$ E. Betzig, G. H. Patterson, R. Sougrat, O. W. Lindwasser, S. Olenych, J. S. Bonifacino, M. W. Davidson, J. Lippincott-Schwartz, and H. F. Hess, Science 313, 1642 (2006).

${ }^{7}$ M. Rust, M. Bates, and X. Zhuang, Nat. Methods 3, 793 (2006).

${ }^{8}$ M. Kuttge, E. J. R. Vesseur, A. F. Koenderink, H. J. Lezec, H. A. Atwater,

F. J. García de Abajo, and A. Polman, Phys. Rev. B 79, 113405 (2009).

${ }^{9}$ F. J. García de Abajo, Rev. Mod. Phys. 82, 209 (2010).

${ }^{10}$ M. Kuttge, F. J. García de Abajo, and A. Polman, Nano Lett. 10, 1537 (2009).

${ }^{11}$ N. Yamamoto, S. Ohtani, and F. J. García de Abajo, Nano Lett. 11, 91 (2011).

${ }^{12}$ T. Suzuki and N. Yamamoto, Opt. Express 17, 23664 (2009).

${ }^{13}$ K. Takeuchi and N. Yamamoto, Opt. Express 19, 12365 (2011).

${ }^{14}$ P. B. Johnson and R. W. Christy, Phys. Rev. B 6, 4370 (1972). 